\title{
Reinvigorating exhausted $T$ cells in hepatitis B infection
}

During chronic infection with hepatitis B virus (HBV), T cells become exhausted, and their antiviral response is weakened. An article in Nature Medicine now shows that reducing mitochondrial dysfunction using mitochondrion-targeted antioxidants in $\mathrm{CD}^{+} \mathrm{T}$ cells from patients with chronic HBV infection can restore $\mathrm{T}$ cell function, which could improve viral clearance.

The current standard of care for patients with chronic HBV infection involves short-term treatment with interferon (IFN) and/or long-term treatment with nucleotide or nucleoside analogues. Recent attempts to reactivate the repressed immune

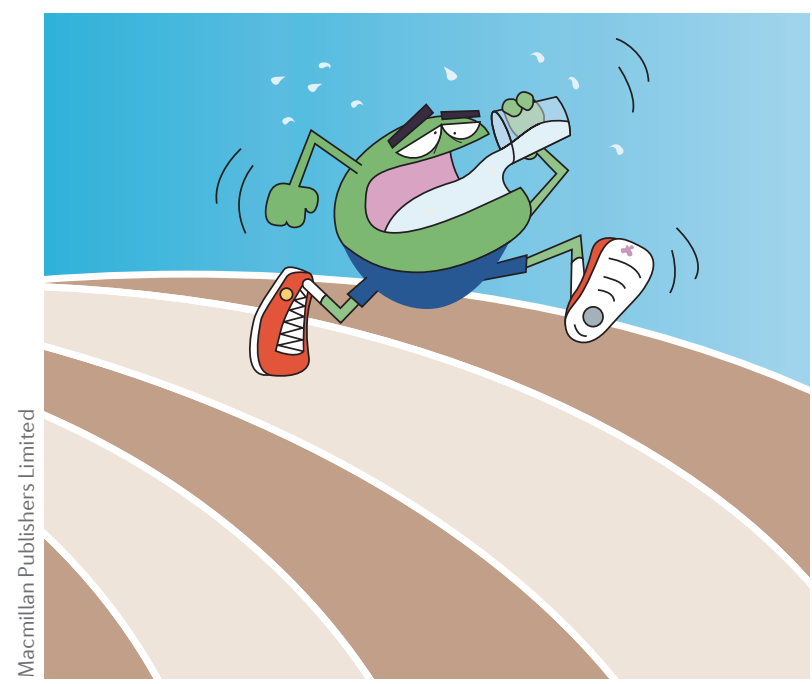

response by relieving suppressive signals, such as those from programmed cell death protein 1 (PD1), regulatory $\mathrm{T}$ cells or immunosuppressive cytokines, have had limited success.

To identify additional mechanisms that could be targeted, Fisicaro and colleagues compared gene expression profiles of HBV-specific $\mathrm{CD} 8^{+} \mathrm{T}$ cells from patients with chronic infection with those from patients whose HBV infections resolved spontaneously. Genes involved in mitochondrial function, proteasome-mediated proteolysis, DNA repair and transcription featured among those that were differentially expressed. Expression of the most markedly downregulated mitochondrion- and proteasomerelated genes was investigated in additional patients; $74 \%$ of these genes were confirmed to be differentially expressed.

$\mathrm{CD}^{+} \mathrm{T}$ cells from patients with chronic HBV infections had reduced mitochondrial polarization and mass biogenesis following stimulation with anti-CD3 antibodies than did cells from patients with resolved infections or healthy individuals. Substantially higher levels of mitochondrial superoxide, which are a measure of reactive oxygen species (ROS) content, were detected in unstimulated cells from patients with chronic infections compared with cells from healthy individuals or those whose HBV infections had resolved.

Altered mitochondrial ROS levels can be corrected with the mitochondrion-targeted antioxidants mitoquinone (MitoQ) or MitoTempo. In peripheral blood mononuclear cells (PBMCs) from patients with chronic HBV infection, these compounds reduced the fraction of $\mathrm{CD} 8^{+} \mathrm{T}$ cells that underwent mitochondrial membrane depolarization following stimulation with HBV peptides.

Importantly, this improved mitochondrial function was accompanied by increased $T$ cell viability and antiviral activity. PBMCs from patients with chronic HBV infection produced more IFN $\gamma$ and/or tumour necrosis factor (TNF) if they were expanded in the presence of one of these mitochondrion-targeted antioxidants. Indeed, levels of IFN $\gamma$ were comparable to those produced by cells from patients whose infections had resolved. The effects of these mitochondria-targeted compounds were greater on $\mathrm{HBV}$-specific exhausted $\mathrm{T}$ cells than on functional $\mathrm{T}$ cells (such as those that recognize influenza virus), which indicates a particular role for restoring mitochondrial function in people with chronic infections.

These data suggest that restoring mitochondrial function in patients with chronic HBV infection could restore $\mathrm{T}$ cell activity and improve viral clearance.

Megan Cully

ORIGINAL ARTICLE Fisicaro, P. et al. Targeting mitochondrial dysfunction can restore antiviral activity of exhausted HBV-specific CD8 T cells in chronic hepatitis B. Nat. Med. 23, 327-336 (2017) 